\title{
BERUFSRISIKO
}

\section{Wie Geschäftsreisen die Gesundheit ruinieren}

Arbeitnehmer, die sehr viel auf Geschäftsreisen sind, haben ein erhöhtes Risiko für gesundheitliche Probleme. Sie bringen mehr Kilos auf die Waage, haben mehr kardiovaskuläre Risikofaktoren und schätzen ihren Gesundheitszustand schlechter ein als Personen, die nur wenige Dienstreisen unternehmen. Das hat eine Auswertung von Daten von rund 13000 US-amerikanischen Arbeitnehmern ergeben.

Im Detail hatten die Vielreisenden (20 Nächte im Monat und mehr) im Vergleich zu Arbeitnehmern mit weniger Dienstreisen (1-6 Nächte im Monat):
- einen höheren BMI (27,5 vs. 26,1 kg/m²),

- ein fast doppelt so hohes Adipositasrisiko,

- ein niedrigeres HDL-Cholesterin (53,3 vs. $56,1 \mathrm{mg} / \mathrm{dl}$ ) und

- einen höheren diastolischen Blutdruck (76,2 vs. $74,6 \mathrm{mmHg}$ ).

Darüber hinaus bewerteten die Vielreisenden ihren Gesundheitszustand 2,6-mal häufiger als mäßig bis schlecht. Diese Angabe gibt Anlass zu Sorge, so die Studienautoren, denn die Einschätzung der eigenen Gesundheit korreliere mit der Mortalität.

J Occup Environ Med 2011;53:358-63

\section{RISIKOFAKTOR FÜR ADIPOSITAS \\ Kleinkind hängt an der Flasche}

Zur Adipositasprävention sollten Kinder nach ihrem ersten Geburtstag vom Fläschchen entwöhnt werden, empfehlen Pädiater aus Cincinnati/Ohio. Sie haben in einer Studie einen Zusammenhang zwischen längerem Gebrauch des Fläschchens und Fettleibigkeit im Vorschulalter gefunden: 22\% von 6750 untersuchten Kindern tranken noch im Alter von

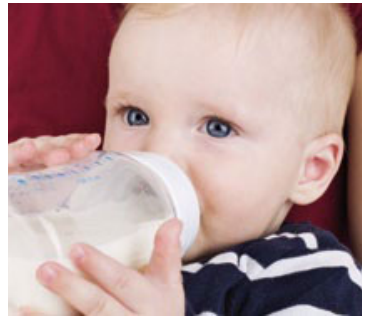
Nach einem
Jahr ist Schluss! zwei Jahren überwiegend aus dem Fläschchen bzw. wurden mit einem Fläschchen mit kalorienhaltigem Inhalt (Milch, Saft) zu Bett gebracht. Diese Kinder hatten ein um 30\% erhöhtes Risiko, mit 5,5 Jahren adipös zu sein $(22,9 \%$ vs. $16,1 \%)$.

Gooze RA et al. J Peds, online 5. Mai 2011; doi: 10.1016/j.peds.2011.02.037

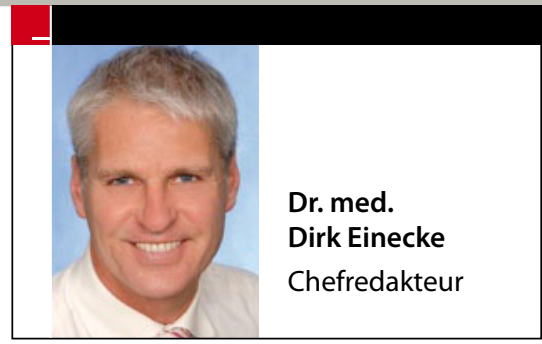

AKUTER HERZINFARKT

\section{Die wichtigsten} Trigger

Forscher aus Belgien und der Schweiz haben die Bedeutung verschiedener Auslöser eines akuten Herzinfarktes verglichen. Wenn sie die Trigger nach ihrer Potenz sortierten, standen auf den Plätzen 1 bis 3 der Konsum von Kokain, schwere Mahlzeiten und Marihuanarauchen. Danach folgten negative Gefühle, körperliche Anstrengung, positive Gefühle, Ärger, sexuelle Aktivität, Straßenverkehr, Atemwegsinfekte, Kaffeetrinken und Luftverschmutzung. Ein ganz anders Bild ergab sich, wenn die Forscher die Prävalenz der möglichen Trigger mitberücksichtigten. Demnach würden auf Bevölkerungsebene die meisten Herzinfarkte ausgelöst durch Verkehrsexposition $(7,4 \%)$, körperliche Anstrengung (6,2\%), Konsum von Alkohol und Kaffee (jeweils $5 \%)$ und Luftverschmutzung (4,8\%). Nawrot TS et al. Lancet 2011;377:732-40

\section{PILZINFEKTIONEN}

\section{Therapieresistenter Aspergillus}

Forscher der Universitäten Manchester und Newark haben eine Zunahme an behandlungsresistenten Aspergillosen beobachtet. Aspergillusinfektionen sind für immunsupprimerte Patienten lebensbedrohlich. In 55\% der Aspergillusproben, die die Forscher untersuchten, fanden sie Marker der Azolresistenz. Mit Azolen (z. B. Itraconazol) werden Aspergillusinfektionen üblicherweise behandelt. Bei einer Studie vor zwei Jahren hatten sie noch eine Resistenzrate von 28\% ermittelt. Besonders beunruhigend: Auch der Aspergillus bei acht Patienten, die nie vorher mit Azolen behandelt worden waren, war resistent gegen das Antimykotikum. Azole werden nicht nur als Antimykotika in der Medizin eingesetzt, sondern auch als Fungizide in der Landwirtschaft. Dies erklärt möglicherweise die rasche $\mathrm{Zu}$ nahme der Resistenzraten.

Clinical Infectious Diseases, 2011 ; 52 (9): 1123 DOI: 10.1093/cid/cir179

\section{www.springermedizin.de}

\section{Neurologie \& Psychiat-} rie für den Hausarzt

Ob Alzheimer, Demenz, Verhaltensstörungen oder Depressionen: Immer häufiger finden sich Patienten mit neurologischen oder psychiatrisch/psychosomatischen Erkrankungen in der Hausarztpraxis. Bleiben Sie auf dem Laufenden: www.springermedizin.de/znshausarztpraxis 